\title{
A regionalidade como determinante do IDE do Brasil: uma análise de dados em painel
}

\author{
Regionality as a determinant of the Brazilian FDI: a panel data analysis
}

\author{
Mariane Santos Françoso'
}

\begin{abstract}
Resumo
Anteriormente, os fluxos de IDE tinham a sua origem fortemente atrelada às economias desenvolvidas. Relatórios recentes de organizações multilaterais têm mostrado que esse fenômeno tem sofrido modificações, com cada vez mais países em desenvolvimento também ocupando importantes posições como grandes realizadores de IDE. A partir dessa consideração, o presente trabalho busca mostrar quais são os determinantes do IDE originado no Brasil e qual o papel da regionalidade para esse processo. Para tanto, é estimado um modelo de dados em painel, com variáveis macroeconômicas e institucionais. Os resultados apontam a não significância da regionalidade e a forte relevância das variáveis macroeconômicas.
\end{abstract}

Palavras-chave: IDE; economias em desenvolvimento; regiões globais

\section{Abstract}

Previously, FDI flows had their origin strongly tied to developed economies. However, recent reports from multilateral organizations have shown that this phenomenon has undergone changes, with some developing countries also occupying important positions as major origins for FDI. From this consideration, the present work seeks to show which are the determinants of FDI originated in Brazil and what is the role of regionality. To do so, a panel data model with macroeconomic and institutional variables will be estimated. The results point to the non-significance of the regionality and the strong relevance of macroeconomic variables.

Keywords: FDI; developing economies; global regions

'Doutoranda em Ciências Econômicas no Instatituto de Economia - Universidade Estadual de Campinas 


\section{Introdução}

Estudos que abordam os determinantes do IDE (Investimento Direto Estrangeiro) têm explorado a dinâmica de tal processo em diversos países, inclusive nas economias em desenvolvimento, que mais recentemente vêm aparecendo também como realizadoras de IDE, e não apenas como receptoras (UNCTAD, 2017). No caso dos países em desenvolvimento, estes são explorados, de forma agregada, em Amal et al (2009); Perez e Nogueira (2016); Stoian e Mohr (2016). Também já foram explorados os determinantes do IDE que os países em desenvolvimento recebem, como em Thangamani et al (2010); Ranjan e Agrawal (2011); Ahmed e Zlate (2014). Contudo, poucos trabalhos exploraram os determinantes do IDE realizado pelos países em desenvolvimento de forma desagregada, alguns exemplos são Gondim et al (2017), Zhang e Daly (2011) e Buckley et al (2007).

Enquanto Gondim et al (2017) e Buckley et al (2007) analisam uma série de variáveis macroeconômicas e institucionais para avaliar os determinantes do IDE realizado pelo Brasil e pela China, respectivamente, Zhang e Daly (2011) analisam também a China, mas se restringem às variáveis de natureza macroeconômica.

Nesse contexto, o presente trabalho pretende contribuir para a literatura que analisa os determinantes do IDE realizado pelos países em desenvolvimento, analisando o caso do IDE originado no Brasil e verificando a importância da regionalidade como um determinante destes investimentos. É dada atenção à variável regionalidade, pois, de acordo com Iammarino e McCann (2016) boa parte dos fluxos de IDE se dá entre países vizinhos em vez de serem realmente transações globais. Como resultado, os países tem se tornado cada vez mais integrados no que é chamado de regiões globais.

Para tanto será replicado o modelo elaborado por Zhang e Daly (2011), com a adição de variáveis de cunho institucional (percepção da integridade do governo e liberdade de realização de negócios). Também é adicionada uma variável binária para os países sul-americanos, a fim de captar o papel da regionalidade como determinante do IDE.

A análise é realizada através de dados coletados em diversas fontes e da análise de dados em painel, com aplicação dos métodos de mínimos quadrados ordinários (MQO) para dados empilhados e de efeitos aleatórios.

Além desta introdução e da conclusão, o trabalho está dividido em mais 3 seções. Na próxima seção é realizada uma revisão bibliográfica teórica e empírica sobre abordagens relevantes e trabalhos já desenvolvidos sobre o tema. Na seção três é detalhada a metodologia aplicada, destacando as variáveis empregadas, sinais esperados e o modelo a ser estimado. Na seção quatro os resultados são discutidos. Finalmente, são feitas as considerações finais.

\section{Revisão bibliográfica}

\section{Determinantes do IDE}

Dunning é um importante autor no que diz respeito à teorização sobre a motivação do IDE (DUNNING 1977; 2000; 2002). O chamado paradigma OLI (ownership, location, internalization), desenvolvido pelo autor, orientou uma série de estudos sobre a motivação e os determinantes do IDE e da internacionalização de empresas (DUNNING, 2000).

O autor considera que a realização do IDE por uma empresa é o resultado da interação de três vantagens: as vantagens de propriedade, de localização e de internalização. As vantagens de propriedade estão relacionadas à propriedade de ativos pela própria empresa, como tecnologia e capacitações gerenciais. As vantagens de localização, cruciais para o desenvolvimento desta análise, têm a ver com as vantagens proporcionadas pela presença da empresa em determinado mercado, como redução de custos produtivos e de transporte e acesso a grandes mercados. Por fim, as vantagens de internalização estão relacionadas às vantagens da internalização de mercados de produtos intermediários internacionalmente. (DUNNING, 2000). Tratando do aspecto localização além do paradigma OLI, Narula e Dunning $(2000,2010)$ afirmam que há uma relação sistemática entre a estrutura, extensão e natureza das atividades das empresas em uma determinada localização e a estrutura econômica do local. Logo, tanto a estrutura local como o IDE recebido interagem em uma via de mão dupla, na qual um impacta o outro.

A forma como as três vantagens vão interagir para a determinação do IDE será muito específica a cada empresa e estará profundamente ligada às condições políticas e econômicas do local em que as empresas estão sediadas e do local em que se pretende investir, à natureza da indústria em que elas estão envolvidas e à estratégia da empresa.

Dunning (2000) afirma que a motivação para a realização do IDE pode ter quatro naturezas: market seeking; resource seeking; efficiency seeking; strategic asset seeking.

No primeiro caso, o IDE é orientado à demanda, visando o acesso a um determinado mercado. No segundo caso, a motivação da empresa é o acesso a insumos. No terceiro caso, ela é motivada pela busca da maior eficiência na divisão de trabalho. Por fim, no último caso, a motivação é baseada na aquisição de ativos estratégicos (DUNNING, 2000).

$\mathrm{O}$ autor ainda afirma que, de acordo com essa análise, é possível prever que alguns países serão mais represen- 
tativos na realização de IDE, devido, entre outros, ao seu histórico, características internas e empresas nacionais. A partir dessa consideração, o autor destaca que países com economias desenvolvidas são mais prováveis realizadores de IDE do que países em desenvolvimento (DUNNING, 2000).

Relatórios recentes da UNCTAD têm mostrado que esse quadro vem mudando nos últimos anos, sendo que algumas economias em desenvolvimento, como China, Rússia e Singapura, estão ocupando importantes posições tanto na atração como na realização de IDE (UNCTAD, 2017).

Outra mudança recente nos estudos relacionados aos determinantes do IDE tem a ver com o entendimento de que os aspectos institucionais também estão envolvidos no paradigma OLI, sendo importantes fatores, principalmente no que diz respeito à localização e internalização, já que a decisão de se instalar em um local e internalizar ou não uma atividade realizada internacionalmente tem a ver também com o ambiente institucional específico ao local em questão, suas leis, estabilidade política e econômica, entre outros (DUNNING E LUNDAN, 2008).

A dimensão institucional está fortemente relacionada com a dimensão geográfica, já que muitas instituições são específicas aos países. A importância dessas duas dimensões é crucial para o entendimento dos determinantes do IDE, principalmente quando tratamos de empresas de países em desenvolvimento que, muitas vezes, ainda estão em etapas iniciais do seu processo de internacionalização, não apresentando posições internacionais tão consolidadas quanto as empresas de países desenvolvidos, que iniciaram esse processo já em meados das décadas de 1970 (BRENNER, 1998).

De acordo com Buckley et al (2007), em diversos estudos que abordam as etapas do processo de internacionalização de empresas de economias em desenvolvimento, como Coreia do Sul, Índia, Argentina, Brasil e Malásia, foi destacado que as operações internacionais das empresas dessas nações foi iniciada em locais próximos ao país de origem, com os quais relações comerciais já tinham sido desenvolvidas e com os quais havia certa proximidade cultural e conhecimento prévio do mercado e de seu ambiente institucional. Essa característica também foi apontada por Iammarino e McCann (2016), que apontam que boa parte dos fluxos de IDE se dá entre países vizinhos, em vez de serem transações realmente globais. Como resultado, os países têm se tornado cada vez mais integrados no que é chamado de regiões globais.

Mais especificamente sobre o caso das empresas brasileiras, alguns estudos já exploraram a questão da internacionalização. Tais estudos mostraram que a internacionalização via IDE é um movimento recente, que se intensificou a partir dos anos 1990 (FRANCISCHINI ET AL, 2015). Uma das principais justificativas para esse movimento tardio é o conturbado cenário econômico das décadas de 1970 e 1980, e a subsequente estabilidade proporcionada pelo controle da inflação e implantação do Plano Real, o que contribuiu para a realização e o planejamento de longo prazo que a realização de IDE requere (GUERRA, 2014).

Quanto aos fatores determinantes desses investimentos, Silva (2003) afirma que os principais determinantes para o IDE das empresas brasileiras é garantir a presença nos mercados externos e ampliar as exportações, gerando economias de escala, que podem impulsionar a competitividade de tais empresas (FRANCISCHINI ET AL, 2015). Hiratuka e Sarti (2011) consideram que, durante a década de 1990, a realização de IDE por parte das empresas brasileiras era motivada, principalmente, pela retração do mercado nacional. Contudo, a melhora nas condições de rentabilidade e financiamento, vividas durante a década de 2000, modificou tal processo, tornando-o mais voltado à valorização das capacidades produtivas.

Quanto aos principais destinos, os estudos verificaram que se destacam os países do Mercosul (Mercado Comum do Sul), seguidos pela Europa e Estados Unidos (HIRATUKA E SARTI, 2011).

\section{Trabalhos empíricos}

Uma gama de trabalhos empíricos vem investigando os determinantes do IDE realizado por economias em desenvolvimento, sendo que alguns incorporaram variáveis institucionais e macroeconômicas, enquanto outros ficaram restritos às variáveis de natureza macroeconômica.

Zhang e Daly (2011) investigaram os determinantes do IDE realizado pela China no período de 2003 a 2009 . Para isso, foi aplicado o método de MQO para dados empilhados, em um modelo composto de uma série de variáveis de natureza macroeconômica, mais uma variável relativa à dotação de recursos naturais, já que, de acordo com os autores, o acesso a recursos naturais é uma das principais motivações do IDE da China. Os resultados obtidos pelos autores mostraram que as exportações, o PIB per capita, o crescimento anual do PIB e o grau de abertura dos países receptores do investimento foram determinantes significativos, influenciando positivamente o IDE, de acordo com o que era esperado.

Também investigando a China, Buckley et al (2007) analisam os determinantes do IDE do país no período que vai de 1984 a 2001. Os autores testaram modelos estimados através do método de MQO para dados empilhados e de efeitos aleatórios, chegando à conclusão de que o segundo era o mais adequado. O modelo estimado pelos 
autores contava com variáveis macroeconômicas e institucionais, incluindo também uma variável de proximidade geográfica, medida através da distância do país de destino do IDE, em relação à China. Os resultados obtidos pelos autores apontaram que o PIB, proximidade cultural e liberalização política eram estatisticamente significantes e apresentavam o sinal esperado.

Gondim et al (2017) analisaram os determinantes do IDE do Brasil, através de uma amostra de 17 países de destino, no período 2001 a 2014. Assim como Zhang e Daly (2011), os autores também aplicaram o método de MQO para dados empilhados, mas em um modelo com variáveis macroeconômicas e institucionais. Adicionalmente, os autores também incluíram uma variável referente aos acordos de investimentos internacionais, assumindo que políticas governamentais também podem afetar a realização de IDE. De acordo com os resultados, as variáveis macroeconômicas foram relevantes para a determinação do IDE do Brasil, sendo que o PIB per capita, abertura econômica e volatilidade do mercado de capitais mostraram-se significativas, enquanto o único aspecto institucional que se mostrou relevante foi a corrupção.

Os modelos estimados nesses três artigos motivaram fortemente o presente trabalho, no qual o modelo construído incorporou elementos dos três artigos citados. O modelo aqui estimado empregou as variáveis macroeconômicas estimadas em Zhang e Daly (2011), variáveis institucionais, como em Buckley et al (2007) e Gondim et al (2017), e uma variável de dimensão geográfica, assim como em Buckley et al (2007).

Assim como em Gondim et al (2017), o presente trabalho usa o IDE realizado pelo Brasil como variável dependente. Entretanto, este trabalho se diferencia ao incluir mais variáveis de cunho macroeconômico, expandir a análise para o ano de 2015, testar tanto o modelo de MQO para dados empilhados como o de efeitos aleatórios, e também incluir uma variável que tenta captar o efeito da regionalidade como determinante do IDE, análise central deste trabalho.

\section{Metodologia e dados}

\section{Seleção de variáveis e elaboração da base de dados}

Como já mencionado, o presente trabalho parte do modelo aplicado por Zhang e Daly (2011) à China. Contudo, como o modelo se baseou apenas em variáveis macroeconômicas, algumas outras variáveis foram adicionadas, a fim de incorporar mais aspectos relevantes à realização do IDE. Assim, o modelo estimado tentou abranger alguns fatores sobre o país de destino do IDE para definir as variáveis independentes, como: a dimensão do mercado, o crescimento dele, a abertura e estabilidade da economia, a taxa de câmbio da moeda do país de destino em relação ao real, burocracia, percepção sobre o nível de corrupção, a já existência de relações econômicas e a regionalidade.

- Dimensão do mercado: o acesso a novos e grandes mercados é uma importante variável para a determinação do IDE, como destacado por Dunning $(1977,2000)$. Para captar esse aspecto será utilizada a variável PIB per capita.

- Crescimento do mercado: também relacionado ao acesso a novos mercados, o crescimento mostra o potencial de continuidade dos ganhos e de aumento do tamanho do mercado. Aqui será usada a variável crescimento do PIB em relação ao período anterior.

- Abertura da economia: mede o quanto a economia é aberta ao capital externo. A abertura aqui é medida pelo IDE recebido/PIB.

- Estabilidade econômica: é medida pela taxa de inflação. Essa medida é importante, pois um ambiente instável contribui para o aumento das incertezas já envolvidas no processo de investimento.

- Taxa de câmbio: medida em relação ao real, é uma importante variável, pois embora um câmbio desvalorizado incentive a exportação, um câmbio valorizado contribui para o IDE. A variável foi calculada individualmente, considerando a cotação de cada moeda local em relação ao real (BUCKEY ET AL, 2007).

- Burocracia: é uma variável de ordem institucional, que tenta captar as dificuldades envolvidas na realização de negócios em um determinado país. Para medi-la foi usado como proxy o índice de liberdade de negócios, estimado pela Heritage Foundation. Esse índice considera aspectos como número de procedimentos, tempo e custos para começar, encerrar e obter licença para um negócio.

- Corrupção: capta a influência da percepção de integridade na governança do Estado. Essa é uma importante variável também de cunho institucional. Para mensurá-la foi usado como proxy o índice de integridade do governo, também 
medido pela Heritage Foundation, que mede o nível de corrupção nos diferentes países. Quanto maior o índice, menor a corrupção.

- Já existência de relações econômicas: é medida pelos valores de exportação e importação entre o Brasil e o país de destino. Essa medida é importante, pois, como afirmado por Buckley et al (2007), países com os quais já existe experiência no estabelecimento de relações econômicas são destinos de investimentos preferíveis aos demais.

- Regionalidade: é uma variável binária, que discrimina os países da América do Sul, atribuindo-lhes o valor de 1, enquanto os outros países de destino tem o valor de 0 .

As variáveis independentes e as fontes de dados estão sumarizadas na tabela 1:

Tabela 1 - Variáveis dependente e independentes

\begin{tabular}{|c|c|c|}
\hline Variável & Medida/proxy & Fonte \\
\hline IDE do Brasil & IDE do Brasil & Banco Central \\
\hline Tamanho do mercado & PIB per capita & World Bank \\
\hline Crescimento do mercado & Crescimento do PIB & World Bank \\
\hline Abertura econômica & IDE/PIB & World Bank \\
\hline Estabilidade econômica & Taxa de inflação & World Bank \\
\hline Câmbio & $\begin{array}{l}\text { Taxa de câmbio considerando- } \\
\text { se o real e a moeda do país de } \\
\text { destino }\end{array}$ & World Bank \\
\hline Burocracia & Índice de liberdade de negócios & Heritage Foundation \\
\hline Corrupção & $\begin{array}{l}\text { Índice de integridade do } \\
\text { governo }\end{array}$ & Heritage Foundation \\
\hline \multirow{2}{*}{$\begin{array}{l}\text { Existência de outras } \\
\text { relações econômicas }\end{array}$} & Exportação & $\begin{array}{c}\text { Ministério da Indústria, Comércio } \\
\text { Exterior e Serviços }\end{array}$ \\
\hline & Importação & $\begin{array}{c}\text { Ministério da Indústria, Comércio } \\
\text { Exterior e Serviços }\end{array}$ \\
\hline Regionalidade & Países da América do Sul & \\
\hline
\end{tabular}

Fonte: Elaboração própria

\section{Modelo estimado e resultados esperados}

A amostra analisada foi composta por 26 países que foram destino do IDE brasileiro de 2006 a 2015 . A análise aqui proposta se inicia em 2006 por esse se tratar do ano em que o IDE realizado por empresas brasileiras passou a desenvolver uma trajetória de crescimento mais estável e por ser o primeiro ano em que o IDE realizado por empresas nacionais superou o IDE recebido por empresas estrangeiras (Hiratuka e Sarti, 2011) . Devido à disponibilidade de dados e à realização do IDE em si, não são todos os países que aparecem em todos os anos, compondo assim um painel desbalanceado (BALTAGI, 2001). Os países que compuseram a amostra são discriminados na tabela 2. 
Tabela 2 - Países de destino que compuseram a amostra

\begin{tabular}{c|c}
\hline Argentina & Luxemburgo \\
Áustria & México \\
Bélgica & Holanda \\
Bolívia & Panamá \\
Canadá & Peru \\
Chile & Portugal \\
China & África do Sul \\
Colômbia & Espanha \\
Dinamarca & Suíça \\
França & Reino Unido \\
Alemanha & Estados Unidos \\
Hungria & Uruguai \\
Itália & Venezuela \\
\hline
\end{tabular}

Fonte: elaboração própria, a partir de dados do Banco Central

O modelo estimado é:

$\operatorname{lnIDE}=\mathrm{PIBpc}+$ crescPIB+abert+infla+txcâmbio+burocracia+integridadegov+exp+imp+AS (1)

Optou-se por estimar o IDE em forma de logaritmo natural a fim de reduzir a variância da variável dependente, já que os valores de investimento vão desde 10 milhões até bilhões de dólares. Esse procedimento também foi adotado por Buckley et al (2007).

O modelo foi estimado através dos métodos de MQO para dados empilhados e efeitos aleatórios, como em Buckley et al (2007) e Stoian e Mohr (2016), verificando, na sequência, qual o método mais adequado.

O método de dados empilhados tem algumas vantagens em relação ao cross section simples, pois possibilita um aumento significativo do número de observações, além de possibilitar a visualização do efeito das variáveis independentes ao longo do tempo (BALTAGI, 2001).

Entretanto, no modelo de dados empilhados não é possível captar o efeito não observado, o que é possível no modelo de efeitos aleatórios, desde que consideremos que ele é não correlacionado às variáveis explicativas (BALTAGI, 2001).

O método de efeitos fixos não será estimado, pois não é adequado, já que a variável central da análise aqui desenvolvida (regionalidade) é constante ao longo do tempo (WOOLDRIDGE, 2006).

As relações esperadas para cada uma das variáveis são mostradas na tabela 3.

Tabela 3 - Sinais esperados para as variáveis independentes

\begin{tabular}{lc}
\hline PIBpc & + \\
crescPIB & + \\
abert & + \\
infla & + \\
txcâmbio & + \\
burocracia & - \\
integridadegov & + \\
exp & + \\
imp & + \\
AS & + \\
\hline
\end{tabular}

Fonte: elaboração própria 


\section{Resultados e discussões}

O modelo econométrico foi estimado pelos métodos de MQO para dados empilhados e efeitos aleatórios, contando com 216 observações. Os resultados das estimações são mostrados na tabela 4.

Para o modelo de dados empilhados foi realizado o teste de Breusch-Pagan/ Cook-Weisberg, para detectar heterocedasticidade (WOOLDRIDGE, 2006). Nesse teste, a hipótese nula de homocedasticidade foi rejeitada. Com isso, optou-se por mostrar os resultados dos estimadores robustos na tabela 4.

Após a estimação do modelo por efeitos aleatórios, foi realizado o teste de Breusch-Pagan. Esse teste detecta a existência ou não de efeitos aleatórios não observados. Caso a hipótese nula (ausência de efeitos não observados) seja rejeitada, recomenda-se o emprego do modelo de efeitos aleatórios, em detrimento do MQO para dados empilhados.

\begin{tabular}{|c|c|c|}
\hline & MQO & $\begin{array}{c}\text { Efeitos } \\
\text { aleatórios }\end{array}$ \\
\hline crescimento do PIB & $\begin{array}{c}0.0059858 \\
(0.0298995)\end{array}$ & $\begin{array}{c}0.0034062 \\
(0.0316705)\end{array}$ \\
\hline PIB per capita & $\begin{array}{c}0.0000173 * * \\
(6.30 \mathrm{E}-06)\end{array}$ & $\begin{array}{c}0.0000319 * * \\
(0.0000125)\end{array}$ \\
\hline Inflação & $\begin{array}{c}0.1311285^{* *} \\
(0.0426544)\end{array}$ & $\begin{array}{c}0.0786769 * * * \\
(0.0400999)\end{array}$ \\
\hline Exportação & $\begin{array}{l}0.000114 * * \\
(0.0000387)\end{array}$ & $\begin{array}{c}0.0000792 * * * \\
(0.0000421)\end{array}$ \\
\hline Importação & $\begin{array}{c}0.0001318^{* *} \\
(0.0000327)\end{array}$ & $\begin{array}{l}-0.0000713 \\
(0.0000448)\end{array}$ \\
\hline Taxa de câmbio & $\begin{array}{c}-0.0004447 * * \\
(0.0000913)\end{array}$ & $\begin{array}{c}-0.0000348^{* * *} \\
(0.0001488)\end{array}$ \\
\hline Regionalidade & $\begin{array}{c}0.5600896 * * * \\
(0.2584932)\end{array}$ & $\begin{array}{l}0.6508387 \\
(0.638252)\end{array}$ \\
\hline Percepção de integridade do governo & $\begin{array}{c}0.0081494 \\
(0.0079658)\end{array}$ & $\begin{array}{l}-0.0123665 \\
(0.0156382)\end{array}$ \\
\hline Liberdade nos negócios & $\begin{array}{c}0.0133207 \\
(0.0139003)\end{array}$ & $\begin{array}{c}0.0204679 \\
(0.0197998)\end{array}$ \\
\hline Abertura & $\begin{array}{c}0.0054079 \\
(0.0059932)\end{array}$ & $\begin{array}{c}-0.000417 \\
(0.0045606)\end{array}$ \\
\hline Constante & $\begin{array}{c}2.474809 \\
(1.105933)\end{array}$ & $\begin{array}{c}2.6738 \\
(1.531456)\end{array}$ \\
\hline $\mathrm{R}^{2}$ & 0.1852 & 0.145 \\
\hline Breusch Pagan & $89.77 * *$ & \\
\hline
\end{tabular}

Nota1: ${ }^{* *}$ Significativo a $1 \% ;{ }^{* * *}$ Significativo a $5 \%$. Nota 2: erro padrão entre parênteses

Fonte: elaboração própria a partir do software Stata 
O teste de Breusch-Pagan rejeitou a hipótese nula de ausência de efeitos não observados, ou seja, os efeitos não observados estão presentes no modelo. Logo, o modelo de efeitos aleatórios é o mais adequado para a estimação do modelo econométrico proposto na equação 1 . Dessa forma, apenas os resultados dessa estimação serão analisados.

$\mathrm{Na}$ estimação por efeitos aleatórios apenas as variáveis de ordem macroeconômica apresentaram significância estatística. Mesmo com alguns trabalhos, como Stoian e Mohr (2016), afirmando que um dos motivos para a realização de IDE por parte de países em desenvolvimento é a tentativa de fugir de problemas institucionais de seu país de origem, as variáveis de cunho institucional (liberdade nos negócios e integridade do governo) não apresentaram significância, não podendo ser consideradas fatores decisivos para o IDE realizado pelo Brasil.

A relevância das variáveis macroeconômicas está de acordo com os determinantes de IDE identificados por Silva (2003). Considerando que o IDE realizado por empresas brasileiras têm forte caráter market seeking, a situação macroeconômica dos países de destino é fundamental para a decisão de consolidar ou não a posição da empresa, através do IDE, em determinados mercados.

Também no caso da regionalidade não foi verificada significância. Embora o Brasil tenha uma relação forte com alguns países da amostra, como Argentina e Uruguai, essa relação é enfraquecida quando olhamos para outros países sul-americanos da amostra, como o Peru. Este resultado também dialoga com o trabalho de Silva (2003), que afirmou que a principal motivação do IDE das empresas brasileiras ainda está vinculada à exportação. Logo, as relações de IDE são mais fortes com os países membros do Mercosul, com quem o Brasil tem acordos de comércio, mas é fraca com outros países da América do Sul, o que impacta na significância da variável como um todo.

Esse aspecto já foi destacado por Souza e Castilho (2016), que identificaram que, embora existam acordos que busquem maior integração na América do Sul e América Latina, estes estão orientados à facilitação dos fluxos de comércio, falhando no que diz respeito a promover maior integração produtiva ou maiores fluxos de IDE entre os países.

As variáveis PIB per capita, exportação e taxa de câmbio foram significativas e mostraram o sinal esperado. Já a variável inflação, embora seja significativa, não apresentou o sinal esperado, mostrando, na verdade, ter efeito positivo sobre o IDE.

De acordo com os estimadores, ceteris paribus, um aumento de 1 dólar no PIB per capita implica em um aumento de $0,003 \%$ no IDE. Um aumento de um dólar na exportação leva a um aumento de $0,008 \%$ no IDE. Já a queda na taxa de câmbio implica em um aumento no IDE, enquanto o aumento de 1 ponto percentual na inflação leva a um aumento de $7,8 \%$ no IDE.

\section{Conclusão}

Mais recentemente alguns países em desenvolvimento têm despontado não apenas como grandes receptores de IDE, mas também como importantes realizadores dele. Esse fenômeno fez com que os estudos que abordam a temática do IDE nesses países fossem além da perspectiva dos determinantes da atração de IDE por parte destes, mas também abordassem os determinantes para a realização de IDE por empresas locais.

Contudo, muitos dos estudos envolvidos nessa temática trataram dos países em desenvolvimento de forma agregada. Atentando a esse aspecto, o presente trabalho buscou contribuir estimando um modelo que analisasse os determinantes do IDE realizado pelo Brasil, em especial o papel da regionalidade, destacado por Iammarino e McCann (2016).

A atenção a esse aspecto deve-se ao fato de que a intensificação do IDE por parte dos países em desenvolvimento é um fenômeno mais recente e estudos já realizados previamente apontaram que empresas em estágios iniciais de internacionalização costumam preferir realizar investimentos em locais próximos.

Com o suporte da literatura teórica e empírica desenvolvida sobre o assunto, foi estimado um modelo econométrico com variáveis de natureza macroeconômica e institucional, além da regionalidade.

Empregando um modelo de efeitos aleatórios, constatou-se que a regionalidade não foi percebida como uma variável significativa na determinação do IDE originado no Brasil, assim como as variáveis de natureza institucional, sendo que apenas as variáveis macroeconômicas mostraram-se estatisticamente significativas. Isso está em linha com o caráter market seeking do IDE realizado pelas empresas brasileiras, o que já foi identificado pela literatura, como em Silva (2003).

Este trabalho oferece perspectivas para trabalhos futuros, pois, embora a regionalidade, aqui determinada com uma variável binária de valor 1 para os países da América do Sul, tenha sido não significativa, é sugerido que esse ponto, que encontra respaldo na literatura, ainda possa ser explorado, buscando-se medidas alternativas ou a investigação de outros aspectos relacionados à ela, como a existência de acordos comerciais. 


\section{Referências}

AHMED, S., \& ZLATE, A. (2014). Capital flows to emerging market economies: A brave new world?. Journal of International Money and Finance, 48, 221-248.

AMAL, M., RABOCH, H., \& TOMIO, B. T. (2009). Strategies and determinants of foreign direct investment (FDI) from developing countries: Case study of Latin America. Latin American Business Review, 10(2-3), 73-94.

BALTAGI, B. H. (2001). Econometrics analysis of panel data. 2 ed. Chichester, UK: Wiley \& Sons.

BRENNER, NEIL. (1998). Global cities, glocal states: global city formation and state territorial restructuring in contemporary Europe. Review of International Political Economy, 5(1), 1-37.

BUCKLEY, P. J., CLEGG, L. J., CROSS, A. R., LIU, X., VOSS, H., ZHENG, P. (2007). The determinants of Chinese outward foreign direct investment. Journal of international business studies, 38(4), 499-518.

DUNNING, J. (1998). Location and the multinational enterprise: a neglected factor, Journal of International Business Studies, 29 (1), 45-66.

DUNNING, J. (1977). Trade, location of economic activity and the MNE: A search for an eclectic approach, in Ohlin, B.; Hesselborn, P.; Wijkman, P. (Eds.) The International Allocation of Economic Activity, London: Macmillan, 395-418.

DUNNING, J. (2000). The eclectic paradigm as an envelope for economic and business theories of MNE activity. International business review, 9(2), 163-190.

DUNNING, J., LUNDAN, S. M. (2008). Institutions and the OLI paradigm of the multinational enterprise. Asia Pacific Journal of Management, 25(4), 573-593.

FRANCISCHINI, A. S. N., FURTADO, J., \& GARCIA, R. (2015). Tecnologia e trajetórias de internacionalização precoce: análise de casos na indústria brasileira. Gest. Prod., São Carlos, 22(2), 267-279.

GUERRA, T. T. A. (2014). Internacionalização de empresas brasileiras: Uma análise macro-setorial da década de ouro (1999 - 2009). Trabalho de conclusão de curso apresentado ao Departamento de Relações Internacionais, Universidade Federal de Roraima, Boa Vista.

GONDiM, I. J. C., MORAndier, N., DiAS, I. R. R., COUTO, C. A. P., CHAROTTA, T. C. A. (2017). Analysis of Domestic Factors Affecting Outward Foreign Direct Investment in Brazil. Latin American Business Review, 18(1), 1-18.

HIRATUKA, C., \& SARTI, F. (2011). Investimento direto e internacionalização de empresas brasileiras no período recente.

IAMMARINO, S., \& MCCANN, P. (2016). Network Geographies and Geographical Networks: Co-dependence and Co-evolution of Multinational Enterprises and Space. In The New Oxford Handbook of Economic Geography.

NARULA, R., \& DUNNING, J. H. (2000). Industrial development, globalization and multinational enterprises: new realities for developing countries. Oxford development studies, 28(2), 141-167.

NARULA, R., \& DUNNING, J. H. (2010). Multinational enterprises, development and globalization: Some clarifications and a research agenda. Oxford Development Studies, 38(3), 263-287.

PEREZ, R., NOGUEIRA, C. (2016). Outward FDI from small developing economies: Firm level strategies and home-country effects. International Journal of Emerging Markets, 11(4), 693-714. 
RANJAN, V., \& AGRAWAL, G. (2011). FDI inflow determinants in BRIC countries: A panel data analysis. International Business Research, 4(4), 255.

SILVA, M. L. (2003). A inserção internacional das grandes empresas nacionais. In M. Laplane, L. Coutinho \& C. Hiratuka (Orgs.), Internacionalização e desenvolvimento da indústria no Brasil (p. 105-163). São Paulo: UNESP.

SOUZA, K. S. G., \& CASTILHO, M. R. Integração produtiva e acordos comerciais: o caso dos países da Aladi. Economia e Sociedade, 25(1), 173-207.

STOIAN, C., MOHR, A. (2016). Outward foreign direct investment from emerging economies: escaping home country regulative voids. International Business Review, 25(5), 1124-1135.

THANGAMANI, B., XU, C., \& ZHONG, C. (2010). Determinants and growth effect of FDI in South Asian economies: Evidence from a panel data analysis. International Business Research, 4(1), 43.

UNCTAD (Conferência das Nações Unidas sobre Comércio e Desenvolvimento) (2017). World Investment Report.

WOOLDRIDGE, J. M. (2006). Introdução à econometria: uma abordagem moderna. Pioneira Thomson Learning.

ZHANG, X., DALY, K. (2011). The determinants of China's outward foreign direct investment. Emerging markets review, 12(4), 389-398.

\section{Anexo 1 - Rotina da análise no Stata}

*descrição dos dados

describe

summarize ide crescpib pibpc infla exporta importa cambio integrigov libernegocios abertura

* gerando variaveis

generate $\operatorname{lnide}=\ln (\mathrm{ide})$

generate expo=exporta/1000

generate impo=importa/1000

*regressão

regress lnide crescpib pibpc infla expo impo cambio as integrigov libernegocios abertura

*distribuição dos residuos

predict e1, resid

kdensity e1, normal

rvfplot

predict resid

predict ychapeu, $\mathrm{xb}$

scatter resid ychapeu

*teste heterocedasticidade

estat imtest, white

hettest

*estimador robusto

quietly regress lnide crescpib pibpc infla expo impo cambio as integrigov libernegocios abertura

${ }^{*}$ Efeitos aleatorios

* codifica categorias nominais em valores numéricos

encode pais, generate(codpais) 
* define unidade de corte transversal (regiao) e temporal (ano) xtset codpais ano

* descreve as características do painel xtdes

${ }^{*}$ regressao efeitos aleatorios completo

xtreg lnide crescpib pibpc infla expo impo cambio as integrigov libernegocios abertura, re est store re

* Teste de breusch Pagan:

xttest0 